\title{
The future of Arms Length Management Organisations: the uncertain fate of a social housing hybrid
}

\author{
Ian Cole* and Ryan Powell \\ Sheffield Hallam University
}

\section{Summary}

The proportion of social housing in England managed by Arms-Length Management Organisations (ALMOs) has increased steadily over the past eight years, until it is now responsible for the management of over one million council homes. Created initially as a conduit for the delivery of the Labour government's Decent Homes programme, the ALMO sector has gathered momentum and acquired a distinctive identity and purpose. This paper discusses how ALMOs are facing up to future challenges in a new political and financial climate, based on a series of interviews undertaken with ALMO Chief Executives at the end of 2009 and in early 2010.

Chief Executives focused on two critical challenges that faced the sector: funding, not least in terms of multiple pressures on revenue budgets, and the quality and maturity of the relationship with the parent local authority. They were keen to retain the broader neighbourhood role that many ALMOs have developed, though this was tempered by the recognition that funding realities might press the organisations back into concentrating on their core housing service. The crux was whether the future financial regime would be amenable for the development of a new style of social housing organisation some where between the current local authority and housing association models. In conclusion, we suggest that some of the organisational, operational and governance arrangements pioneered by ALMOs seem to within the coalition government's enthusiasm for organisational autonomy, localism and empowerment. This may offer an opportunity for ALMOs if, and only if, the financial sums can be made to add up to ensure their survival.

Keywords: social housing, Arms Length Management Organisations (ALMOs), Decent Homes, localism.

\section{Arms Length Management Organisations in Housing}

The social housing sector in England has undergone significant change in recent years as a series of measures affecting governance, finance and management have been implemented by central and local government. The election of the Coalition 
government in May 2010 presages an even more dramatic period of change for the sector in the context of the most substantial reductions in public expenditure for more than a generation. This report focuses on the Arms Length Management Organisation(ALMO) sector and the way in which ALMOs are responding to new developments and increased pressures on their resources and the potential consequences for governance and financial arrangements.

The first ALMOs were launched in 2002 and there are now 70 organisations in 66 local authorities managing over one million council homes (NFA, 2010a). Collectively ALMOs have invested in excess of $£ 4$ billion in bringing over 250,000 properties up to the Decent Homes standard (NFA, 2010b). ALMOs are characterised by a strong commitment to tenant involvement empowerment, strong links with their local area, a fixed geographical focus, and an emphasis on close partnership working with local authorities and other stakeholders. In order to secure Decent Homes funding for investment, ALMOs have to achieve at least a two star rating in inspections by the Audit Commission. By the end of 2009, 30 ALMOs had achieved a two star rating and a further 20 had been given the highest, three star, rating - a profile that compares very favourably with housing association equivalents.

Some housing measures are announced with a fanfare - a ministerial statement, a major piece of legislation, a radical change of direction by a new administration. Others enter the arena more surreptitiously, and develop incrementally from small beginnings, so their significance only becomes apparent some time later. The development of the ALMO sector definitely falls into the latter camp. Initially ALMOs were devised (or, perhaps more accurately, improvised) to provide an alternative option for those local authorities that had decided not to transfer stock to a housing association (whether as a result of a tenant vote against transfer or not) in order to free up opportunities for additional borrowing and investment to meet the objectives of achieving the 'Decent Homes' Standard ${ }^{1}$ for all social housing stock by the end of 2010.

Local authorities could apply to central government to set up an arms length management organisation which was then subject to an inspection by the Audit Commission, and assessed according to a three star rating. Those that achieved at least a two start rating (either from the outset or in a subsequent inspection) could apply for additional funding from the Communities and Local Government Department, and this could be invested in its Decent Homes programme. This funding was made available for a specific period. Decent Homes funding was provided for qualifying ALMOs over six annual 'rounds'.

The raison d'être of AMOs was therefore linked to the delivery of the Decent Homes programme, a specific and time limited initiative. This raises the question about what happens when 100 per cent of homes in any locality reach the decency standard. Do ALMOs revert to their former status or metamorphose into a new type of landlord organisation altogether? Or will more local authorities be tempted to review the prospects for transferring the whole stock to a housing association? The role of the Audit Commission in undertaking regular inspections of ALMO performance was also unusual. The overall improvement noted in ALMO ratings by the Audit Commission has provided some independent testimony of the advances that have been made. However, some advocates have suggested that these improvements (in areas such as tenant involvement, 'housing plus' work or business planning practices) might be placed at risk if they were to revert to their original position as 'conventional' council housing.

However, while individual ALMOs have been subject to a regime of regular inspections, there has been relatively little independent evaluation of the sector as 
whole - its evolution, its impact, and its future development. This modest study is a small step to remedy this gap in research. Interviews were undertaken with 17 ALMO Chief Executives. We would be the first to acknowledge the modest nature of this research exercise. It only covers the views of Chief Executives, and not tenants, Board members or other key stakeholders and of course there is likely to be considerable diversity of views about the way forward for any ALMO. There is inevitably a great deal of tentative judgement in what follows.

A key factor in the future for ALMOs lies in the imminent decision to be made on the reform of the housing finance system for council housing. The previous Labour government launched a review of the Housing Revenue Account system in 2009. This process, at heart, involves examining the potential for redistributing housing debt across all those local authorities in England which have retained, rather than transferred, their housing stock (whether managed directly by the council or through an ALMO). Once this one-off redistribution is made (so that those landlords currently in surplus carry some of the debt burden of other councils) local authorities would then operate the housing service on a self-financing basis, setting business plans for a thirty year period so that the revenue stream from rents would be able to service ongoing debt, any additional borrowing for future investment, and ongoing management and maintenance costs (Communities and Local Government, 2010).

These potential reforms offer both an opportunity and a threat to ALMOs. On the one hand, a self- financing future would enable ALMOs to take fuller control over decisions at a local level, without being dependent on annual subsidy determinations handed down to them by central government. It would also enable them to plan for the long-term, making apparent any necessary assumptions about future rent levels, ongoing costs and investment priorities. On the other hand, self-financing places the viability of ALMOs centre stage and much will depend on the terms of the local financial settlement if HRA reform is undertaken. Concerns have been expressed that an approach which would make only limited allowances for management, maintenance and major repairs might make the HRA unviable in the medium to long term. The only prospect then would be to increase rental income or to reduce the level of service to tenants or scale down ongoing maintenance programmes. It can be seen that the outcome of any HRA reform are more likely to have as much to do with arithmetic as policy principle.

\section{Findings}

The research was intended to provide a qualitative assessment of the main issues facing ALMOs in the short and medium term, focusing on four key areas:

- the general performance of the ALMO sector and progress that has been made to date

- the main challenges facing the sector

- options for how ALMOs might respond to these challenges

- key messages Chief Executives would like to relay to central government policymakers about the development of the sector.

Interviews were very much focused on the immediate future and the key issues identified by respondents. This, however, inevitably led onto discussions and reflection regarding the relative performance of ALMOs over their short history, both individually as an organisation and collectively as a housing sub-sector. The relative performance 
of ALMOs was, for many respondents, a key factor in shaping consideration of future options as well as the relationship with the strategic housing authority.

Although there has been no comprehensive research evaluation of the ALMO sector since the emergence of this new organisational structure in 2002, official inspections, anecdotal evidence and political and media discourse suggest that ALMOs have been relatively successful in delivering on their objectives. On the whole they are seen to have met their objectives on Decent Homes and tenant empowerment, and have often played a significant yet developing role in the wider regeneration of their local areas.

Given that the original role and purpose of ALMOs was integrally linked to the delivery of the Decent Homes programme, respondents were asked to summarise their progress in meeting their respective target. In the majority of cases this target had either been met, was close to being met, or was on course to be met (see NFA, 2010b). On the whole however the programme had been (or was being) delivered to schedule, and high levels of satisfaction were reported, both from tenants and from local authorities (see CRESR (2010) for some confirmation of this view).

As a result of this progress, some felt that the initial rationale for the creation of ALMOs had run its course. One interviewee thought that ALMOs had been successful because "there was a clear rationale" from the outset, which was about improving tenant involvement, driving up standards and then securing funding to maintain and continue improving those standards. However, some respondents felt that there was not a clear game plan after the Decent Homes programme was completed and several felt that there needed to be a decision from central government either to encourage ALMOs to play a wider role in the regeneration of their local areas or to bring them 'back in-house'.

There was acknowledgement that the 'ALMO concept' was not that widely understood outside the housing sector and few people knew "what an ALMO actually does" or how it relates to the local council. In this respect the acronym ALMO was considered unhelpful:

"I think the big difficulty is selling the concept of ALMOs, I think that's one of the things that six or seven years on I think people are still struggling to understand, and a horrible acronym, arms length....most people know what an RSL is and what it does and how it operates but I think a lot of people still struggle with ALMOs."

A critical concern for many, however, was in maintaining the Decent Homes standard across the stock and not losing the gains that had been won so far. Whether perceived as an opportunity or a lack of direction and purpose, reflections on the role, purpose and image of the ALMO sector confirm that it was at a critical juncture in its development.

Regardless of future organisational changes and arrangements, there was universal acknowledgement of the legacy from ALMOs that could be built upon in terms of tenant empowerment and governance, as these factors have been at the forefront of the development of the ALMO model. Most respondents reported that some change to governance arrangements had taken place since the ALMO was first formed, with efforts to increase tenant engagement being the key driver of change.

Around half had conducted some kind of governance review which typically reviewed the composition of the Board, the breadth of involvement, and the effectiveness of various area panels, committees and sub-committees that had been set up. While there were local differences in terms of the remit and function of the various committees, most alterations to governance arrangements had been relatively 
minor. Renewal of Board membership was common - with turnover greatest among councillors - and several ALMOs had reduced the size of their Board to make it more manageable (typically from 15 to 12). Most, however, had maintained the "conventional" equal split on the Board between tenants, councillors and independent representatives.

Aside from making pragmatic changes to improve operational effectiveness, the primary motivation for reviewing governance was to increase tenant involvement and control. In many cases, as the ALMO had matured and developed, tenants had taken on a more significant role in setting local standards and in making decisions on investment priorities. This was most evident among ALMOs that had met (or were well on the way to meeting) their Decent Homes target. Increased tenant involvement and leadership was seen as a natural and important progression in the evolution of the organisation. Several Chief Executives reported an evolution of a more tenant-led approach. This was evidenced in the increasing numbers of tenants on the Board and the growth in tenant involvement on all aspects of service delivery. Typically in these cases a conscious decision had been made to make tenants the largest group on the Board.

Tenant involvement within the ALMO sector was seen by the majority as a clear area of strength and a justification in itself for this organisational model. Where previously the tenant participation agenda had "felt like lip service" with the locus of decisionmaking remaining in town halls, it was claimed that there was now a sense of real empowerment for tenants. The breadth and depth of involvement among tenants had undoubtedly improved, but it was acknowledged that there were still formidable obstacles to overcome in terms of engaging traditionally 'hard-to-reach' groups such as young people and minority ethnic communities. Nevertheless, on balance relations with tenants, tenant involvement and tenant satisfaction had been areas of real progress, which many put down to the singular local focus of ALMOs which was contrasted favourably to the more disparate geographical operation of many RSLs. As one respondent put it:

"On the tenant engagement side of it there's a much closer link to tenants and leaseholders...the ALMOs are really focused on what they do locally as opposed to a housing association with property all over the country. I think that's a real strength within the ALMO sector."

Without exception, respondents felt that tenants were now more empowered than before, to the extent that it would be very difficult to reverse some of these trends, whatever form the future housing service took. This is exemplified by the following view.

"I think our residents here will be really reluctant to let go of the current model, on some things they actually oversee the procurement of contracts, they oversee the lettering of those, they oversee staffing structures, the way in which we shape and deliver services."

Respondents also felt that the 'culture of openness' within the sector had contributed to good governance and helped to drive up standards. Many respondents spoke of the positive experiences of sharing best practice and information, collaboration and support within the ALMO sector. The National Federation of ALMOs (NFA) has tried to promote a culture of collaboration and knowledge, especially through encouraging two and three star ALMOs to engage with colleagues elsewhere in organisations that were not performing as well. 
This collaboration was deemed to be a significant difference between ALMOs and both retained stock councils and RSLs. Given their bounded geographical focus, ALMOs were considered to be less competitive with each other than either local authorities, where the potential for collaboration could be undermined by political differences, or RSLs, often in direct competition with one another for development funding.

When asked about the main challenges for their ALMO, two issues predominated: finance and revenue problems (and, as a consequence, maintaining the Decent Homes standard); and the quality of the relationship with the local authority. Uncertainty over any future subsidies, reductions in management fees, and a general squeeze on revenue as a result of the economic downturn meant that the future financial position of ALMOs was, in most cases, rather precarious. This would have obvious implications for the future provision of services and standards and placed a question mark over the continuing viability of many ALMOs. There were two key areas of frustration: the potential for declining standards after a great deal of effort had been expended in driving them up; and the paradoxical situation where ALMOs were delivering to their objectives and were highly regarded, yet were nonetheless under threat.

Those aspects of the service most at risk were the more innovative initiatives and schemes falling outside statutory obligations which had helped ALMOs make a distinctive contribution as broadly based landlords. The majority of ALMOs had met (or were about to meet) their Decent Homes target, but significant challenges remained for those ALMOs which had been approved for the later funding rounds. Many of these organisations cited maintaining decency as a significant challenge in its own right. In a few years time the number of non-decent properties would be rising again and in some cases "there will be whole estates going non-decent". The long-term future was daunting and, while many respondents were fairly optimistic about the short-term financial position, the outlook beyond that was characterised by uncertainty.

At the time of interview, some hope was expressed that the imminent HRA review would secure a more sustainable financial future for ALMOs. However there was also considerable scepticism about the likely outcome, and a minority of respondents thought that the only option to ensure future funding for the services was through the stock transfer route, especially if self-financing was only going to guarantee the 'basic' Decent Homes standard. This view of course assumed that there would be tenant and local authority support for transfer, and an appetite among RSLs (or an 'in house' body) to take control of the stock. This is not a foregone conclusion if transitional financial support is not forthcoming from central government.

Given the difficult financial context in which ALMOs will be operating in the future, there was some disquiet about the apparently contradictory nature of the (then) policy framework. ALMOs were being guided by various government priorities but respondents felt that what was being asked of ALMOs on a national level was inconsistent with the financial realities on issues such as meeting environmental targets. Some respondents were seeking to off-set the impact of the harsher financial climate by engaging early with local authorities and their tenants to allow an open debate on the choices to be made over the future level of services.

The level of uncertainty around future finance was especially problematic for those respondents who were keen to plan ahead to ensure that they were in an appropriate position, organisationally and structurally, to take advantage of potential future investment opportunities. This was becoming increasingly difficult, certainly until the policy parameters for the future of social housing were clearer.

The future organisational structure of the housing service, and therefore the fate of ALMOs, was seen as a decision to be taken by the local authority. Many respondents 
were confident in the ability of their organisation to expand its activities, but it was recognised that any enhanced role was dependent on the position of the local authority. For many respondents, maintaining a partnership approach was the overriding consideration, given the fact that the local authority continued to own the housing stock, and determined the management fee for the ALMO. In a minority of cases, however, partnership working between the local authority and the ALMO was less successful.

In terms of the future, most respondents felt they enjoyed a good working relationship with their respective local authority and saw little reason why this should change. Others felt that a strain in the relationship was inevitable given the financial climate and the pressures placed upon local government to make savings which would inevitably be passed on to the ALMO. One respondent felt that there was potential for conflict in such cases, especially if the council then wanted more control over what the ALMO was doing (and spending). The level of autonomy for ALMOs felt that the original principle (as they saw it) of them operating as semi- autonomous agencies had not materialised.

The desire for more autonomy for ALMOs was regularly raised and this was further complicated by the perceived role at that time of the Tenant Services Authority and the future of regulation (the interviews were undertaken before the recent announcement from the current government about the probable abolition of the TSA and the incorporation of its regulatory functions by the HCA and Housing Ombudsman). The key issue here was the need for different ALMOs to provide a broadly comparable set of standards, albeit focused on local needs. Several respondents expressed frustration about this process, which was considered symptomatic of the tendency for policy initiatives and guidance to be "removed from financial realities". At present the statutory framework also suggests that the local authority rather than the ALMO is the focus of regulatory activity but that distinction is easier to draw in theory than in practice. Respondents felt that the close relationship between the ALMOs and local residents meant that they needed to be included as significant stakeholders in their own right.

\section{Options for the Way Ahead}

Given the extent of uncertainty outlined above and the imminent HRA review it was difficult for respondents to look ahead into the future. Even where Chief Executives felt that there was a common position between the ALMO and the local authority on what should happen, they were reluctant to rule out other possibilities, as the following quote illustrates:

“I don't think there's any appetite for transfer. I think people see that we've been sufficiently successful and low risk not to want to drag us back into the council. I feel reasonably confident about saying that but l'm not at all complacent about it because you just don't know what's going to happen."

Most ALMOs were in continuous dialogue with their respective local authorities about the options available to them, which came down to three possible scenarios:

- continuing with the ALMO model, possibly in a 'reformed' organisational and funding regime

- stock transfer

- moving the housing service back 'in-house'. 
Some respondents stated that a formal review of the housing service had been undertaken or was being undertaken, often as part of a formal option appraisal. Most, however, were awaiting the outcome of the HRA review (and indeed the general election) before commencing a comprehensive review of the service. (For more information about the different options for the future development of ALMOs, see NFA, 2009). There was little appetite at the time of interview for a community ownership approach such as a transition to a Tenant Management Organisation (TMO) or Community Land Trust (CLT). Such options however, were not being entirely ruled out for the future. It was clear from respondents that decisions on the future of the housing service would be made primarily on the basis of the projected financial position once the outcome of the HRA review was known.

Perhaps unsurprisingly, given the performance of the ALMO sector and the positive commitment to this structure and form of governance, the popular preference was to maintain the ALMO model - albeit under a different financial regime which could guarantee the funding to maintain, and preferably extend, the Decent Home standard. It should also allow the ALMO model to develop from its core housing service function to become a wider vehicle for regeneration and neighbourhood management.

There was a degree of optimism about the maintenance of the ALMO ethos and principles of localism, accountability and a strong commitment to tenant involvement and democratic processes. In this respect the values which had been instilled into the housing service through the ALMO were viewed as a positive legacy to be retained regardless of the future organisational structure and status of the landlord. Other ALMOs had not yet entered into formal conversation with their local authorities about options for alternative models of housing management and delivery, but doors were being left open.

One respondent stated that they had completed a stock condition survey and the results were "fine", which provided further reassurance that there was no desperate need to "rush down the LSVT route". However, for many other ALMOs in a relatively worse financial position stock transfer was an increasingly attractive option:

“We've had financial difficulties, so for us improving the service has very much been about maintaining basic services, not doing a lot of fancy stuff...Having appropriate levels of revenue in order to deliver services is really quite important for us. Or if the investment in the stock and the revenue to manage the service are not forthcoming, then for the future it looks increasingly then at solutions that are about more and more partial stock transfers".

The most improbable scenario for the Chief Executives was the housing service moving back in-house and under the control of the local authority. Although this process was unfolding in the London Boroughs of Ealing and Hillingdon at the time of our interviews, most respondents were fairly confident that this would not happen in their locality. It was generally felt that the achievements of the ALMO would not have been matched had the service stayed in-house and could even be lost were the housing service to revert back.

Of course, a pinch of salt might be a necessary complement to these judgements, as ALMO Chief Executives might be unlikely to launch into fundamental critiques of the sector they work in and the organisations they lead. But it should be noted that the progress ALMOs have made has been recognised by more independent voices, as shown in evaluations of policies such as Decent Homes and tenant participation compacts (CRESR, 2009). 
As stated earlier, many ALMOs have diversified their activities to take on more responsibilities above and beyond the delivery of the core housing service and the Decent Homes programme. Chief Executives judged this development to have been successful in bringing wider benefits to estates and neighbourhoods. It was therefore difficult for some to envisage a retrenchment to delivering just a core service in the face of daunting financial pressures. The following quote is typical:

"We would definitely focus our limited resources on basic frontline services to make sure that we're making improvements and saving money and delivering on quality rather than on some of these other 'nice to dos'. But I also think that you can't avoid being part of those neighbourhood management solutions for very long because you just end up with estates that people don't want to live on and then you end up with the management problems anyway".

The stage in the ALMO's development was also relevant here. For those still delivering the Decent Homes programme it made sense that this should be the priority. For those ALMOs that were already performing a wider role there was a sense that this was a natural progression and that the landlord's identity was intimately related to providing these additional services.

For the more mature ALMOs, where Decent Homes was complete and where additional services were already being delivered, there was a view that taking on a wider role (aligned with existing structures and organisation) could influence the council's decision on the future of the ALMO. More ALMOs were getting involved in complementary policy agendas on fuel poverty, climate change, worklessness, reducing overcrowding, providing better family homes, new build etc - which could make a crucial contribution to estate regeneration in the future (For further details see NFA, 2010b). One respondent stated that if ALMOs performed particularly well above and beyond the core service, local authorities might be less inclined to seek efficiency savings and bring them in-house, or pursue alternative options. This was also influenced by the balance to be struck between short term pressures on funding and a longer term perspective on community sustainability, which would highlight the potential benefits of ALMOs delivering more services.

A key factor behind ALMOs adopting a broader remit was the existing positive relationship between tenants and the landlord as a result of significant service improvements after "years of mediocrity". Improving the core housing service facilitated the development of respect and trust and lay the ground for tenant engagement in neighbourhood management and regeneration. Most mature ALMOs had evolved into something more than providers of housing management and maintenance and they did not want to turn back the clock unless it was totally unavoidable.

Many Chief Executives felt that ALMOs collectively needed to be more assertive about the progress that had been made and the overall strengths of the sector - not least, its local orientation, the commitment to partnership working and the progress in offering genuine opportunities for tenant empowerment. The majority of respondents considered that the successes achieved in the sector had been underplayed. While the National Federation of ALMOs was praised for its role in raising awareness about the sector and highlighting achievements, some respondents felt that more should be done on a collective basis.

In national policy terms, there was a view that housing affordability (and delivering new housing supply) had risen up the agenda since the onset of the recession, at the expense of issues such as local democracy, community empowerment and customer service. These are areas where ALMOs tend to perform well and are well placed to 
deliver on objectives, so it was perhaps not surprising that respondents felt that achievements in these spheres had not been as widely recognised nationally as they should have been.

Another recurrent message for policy-makers to pay heed to was the need for more flexibility over regulation, monitoring and review. Many respondents felt that the burden of external monitoring and review, and the bureaucratic processes that accompanied it, were a drain on their resources. The predominant view was that central government needed to place more trust in local authorities to manage their own services which would, in turn, allow ALMOs to operate in more flexible and innovative ways. Several respondents felt that ALMOs had the potential to become influential social entrepreneurs and were well positioned to engage in community-based activities on issues such as health, employment support, training and development. Current constraints on ALMOs' mode of operation would have to be loosened if this potential were to be fully realised.

All the Chief Executives interviewed thought that the housing service they were operating now was an improvement on what existed prior to the emergence of their respective ALMOs. Several factors attributable to this success were regularly mentioned, including:

- a performance orientated culture with a track record of large scale delivery

- a culture of sharing information and knowledge which had helped drive up housing management standards, with some improvements being brought to bear on the RSL sector too

- a local area-based focus ensuring a 'rootedness' to place

- a closer working relationship with tenants and leaseholders and better communication leading to genuine tenant engagement, empowerment and organisational accountability

- an organisational structure and record of engagement which is well suited to a wider role in neighbourhood management and regeneration with local empowerment at the core.

\section{Conclusions}

At the time of writing there has not been a definitive public statement by the new Government on the future prospects for the ALMO sector. Grant Shapps, the Housing Minister, has gone on record by acknowledging that ALMOs 'have done a good job', but without providing more clues about how they might develop. Nevertheless, it is worth pausing to consider the potential impact of statements that have been made so far about the government's policy priorities that will have an important bearing on the context in which ALMOs will be working in the immediate future.

The future financial pressures on social housing organisations and the tenants in the sector will inevitably be immense given the scale of the planned reductions in public expenditure. This may threaten the viability of a broader 'social' model of landlordism, the upkeep of the housing stock to existing standards, the capacity to meet new energy efficiency targets and, above all, the ability of some tenants in acute social and economic need to remain in the sector at all.

The most pressing decision affecting ALMOs concerns the fate of the proposals for the reform of the Housing Revenue Account. The new government continued the consultation exercise on the proposals for HRA reform set in train by the previous 
administration and a decision is expected imminently. The announcement in the 'emergency budget' of cutbacks in housing benefit, and the cap on rent levels, will clearly impact on the tenants and the revenue position of all ALMOs, especially those in higher cost housing markets.

On investment, the government has already cut back the funding of the Homes and Communities Agency (HCA) and is set to recast its role to operate more as an advisory body working with local authorities and other stakeholders to develop funding sources and deliver schemes. The overall prospect for public expenditure is of course very bleak, with cutbacks of up to 40 percent likely in the CLG's budget over the next five years - the precise implications of this for functions and services will only become clearer with the Spending Review announcement in October. In advance of that, local authorities are already planning to make substantial cuts in their budgets in the light of the government's decision to freeze council tax for 2011/12 and 2012/13.

In terms of regulation, it appears that many of the functions of the Tenants Services Authority are to be transferred to the HCA and to the Housing Ombudsman, though it is unclear at the moment whether the TSA itself would survive as an entity. The Audit Commission's Comprehensive Area Assessments have been abolished, and other components of their regulatory role are likely to diminish as well.

Where will all this leave ALMOs? It is worth separating out the financial exigencies they will have to deal with from the operational and governance principles that have underpinned the development of the sector so far. One does not have to be Cassandra to suggest that the financial prospects for ALMOs will be extremely difficult. A degree of certainty on the future of housing revenue will enable ALMOs and their parent local authorities to develop business plans with fewer caveats and uncertainties - but the pressure on rent levels, to maintain decency in the medium to long-term, while avoiding pricing out those households reliant on housing benefit, will be immense. Any complementary activity on neighbourhood management, community involvement, carbon reduction or skills development - the wider role dear to the heart of many tenants and officers in the ALMO sector - is likely to come from judicious use of other funding sources, not least from the private and third sectors, rather than the organisation's own resources.

On capital investment, beyond steady state maintenance and replacement it is difficult to imagine ALMOs and their local authorities being able to go the extra mile (including meeting any enhanced Decent Homes standards). The way ahead will again lie in developing effective partnerships with other stakeholders (where ALMOs' experience of dealing with contractors, for example, over procurement and delivery for Decent Homes could be invaluable). Even on an optimistic scenario, it is likely to be some time before market conditions are sufficiently favourable to support much activity of this kind. And in more socially and economically deprived communities, often with the most pressing investment needs, any economic upswing is likely to be patchy and belated at best.

So far, so bleak. But there are several attributes of ALMOs in terms of their governance and operational principles that may give more reason for optimism about their future. The 'ALMO model' is basically an improvised hybrid between the council and RSL sector, though of course the local authority retains ownership of the stock. Depending on changes to funding, there may be opportunities for ALMOs to consolidate their position as an independent 'third arm' of social housing, operating as social entrepreneurs and fully incorporating principles of mutuality into their governance and accountability structures. The recent ministerial announcement placing great emphasis on developing foundation trusts within the National Health Service may be relevant here, as a potential model that could be adapted. The undoubtedly strong 
track record of ALMOs on tenant involvement also speaks directly to the Coalition Government's concerns to develop modes of community empowerment alongside this.

Finally, the fact that ALMOs have strong local roots and clear geographical boundaries to their operations also fits within the government's declared commitment to localism and to redistribute resources (and responsibility) from larger national, regional or sub-regional bodies and strategic agencies to those organisations 'delivering' at the front line. Of course, translating principles into a viable future organisational model is far from straightforward. It will be a tricky path to tread. But the evidence from this survey of ALMO Chief Executives is that there is a strong desire to preserve some of the guiding principles behind ALMOs - on community involvement, flexibility of approach, a shared ethos, and a neighbourhood focus - which may be threatened by reversion to council retained stock or, more probably, stock transfer to an RSL. It is a 'third way' in social housing that the current administration may be keen to see develop, despite what it might consider to be the rather unfortunate connotations of that term in recent history.

* Correspondence Address: Ian Cole, Centre for Regional Economic and Social Research, Sheffield Hallam University, Howard Street, Sheffield, S1 1WB. Email: i.d.cole@shu.ac.uk.

\section{Notes}

1 The Decent Homes Standard (DHS) was set up to ensure that all homes meeting the minimum requirement would be warm, wind- and weather-tight and with reasonably modern facilities. The initial standard was revised in 2002 and again in 2006 . The DHS is comprised of four criteria. First, it must meet the statutory minimum standard for housing, and be free of category 1 hazards as set out in the HHSRS. Second, it should have reasonably modern facilities and services, in terms of kitchen and bathroom facilities, external noise insulation and common entrance areas for blocks of flats. Third, it should be in a reasonable state of repair. Fourth, it should provide a reasonable degree of thermal comfort, with efficient heating and effective insulation.

This paper is an abridged version of the Research Paper ALMOs at the Crossroads but which Way Home? by lan Cole and Ryan Powell and available from CRESR, City Campus, Sheffield Hallam University Sheffield S1 1WB.

\section{References}

Communities and Local Government (2010) Council Housing: A Real Future. Consultation Paper. London: Communities and Local Government.

CRESR (2009) A Review of Tenant Participation Compacts. Research report to the Department of Communities and Local Government. Available from CRESR, Sheffield Hallam University, Sheffield S1 1WB.

CRESR (2010) An Assessment of the Decent Homes Programme (to be published by the Department of Communities and Local Government later in 2010).

NFA (2009) A Future for ALMOs - Within Local Communities. York: National Federation of ALMOs.

NFA (2010a) Annual Review 2009/10. York: National Federation of ALMOs.

NFA (2010b) Decent Homes, Thriving Communities, Brighter Futures. York: National Federation of ALMOs. 